\title{
Pedestrian Group-Crossing Behavior Modeling and Simulation Based on Multidimensional Dirty Faces Game
}

\author{
Shunqiang Ye, ${ }^{1,2}$ Lu Wang, ${ }^{1}$ Kang Hao Cheong, ${ }^{3}$ and Nenggang Xie ${ }^{1}$ \\ ${ }^{1}$ Department of Mechanical Engineering, Anhui University of Technology, Anhui, Ma'anshan 243002, China \\ ${ }^{2}$ College of Management and Economics, Tianjin University, Tianjin 300072, China \\ ${ }^{3}$ Engineering Cluster, Singapore Institute of Technology, 10 Dover Drive, Singapore 138683 \\ Correspondence should be addressed to Lu Wang; wangluahut@163.com
}

Received 23 June 2017; Accepted 26 September 2017; Published 12 December 2017

Academic Editor: Roberto Natella

Copyright (C) 2017 Shunqiang Ye et al. This is an open access article distributed under the Creative Commons Attribution License, which permits unrestricted use, distribution, and reproduction in any medium, provided the original work is properly cited.

\begin{abstract}
The conflict between pedestrians and vehicles plays a significant role in influencing the efficiency of intersections. In turn, the effectiveness of intersections greatly affects the entire network. Statistical data indicates that up to $70 \%$ of people move in groups (such as friends, couples, or families walking together). The pedestrian group-crossing behavior, as well as an analysis of the dynamics between groups of pedestrians and vehicles at unsignalized intersections, deserves a thorough study. In this paper, a model based on the multidimensional dirty faces game is proposed to analyze the crossing behavior of pedestrians and vehicles as "rational people." Computer simulations were performed to investigate the effect of the group size on crossing behavior and conflict risks. The relationship between heterogeneity of waiting time and walking speed is also investigated. These findings can be used to advance understanding on the "Chinese style road crossing" phenomenon and elucidate the dynamics involved.
\end{abstract}

\section{Introduction}

Pedestrians are among the most vulnerable road users. Traffic accidents involving pedestrians have become a major problem, especially in developing countries $[1,2]$. According to a WHO's Global status report on road safety, more than 1.2 million people die each year as a result of road traffic crashes and pedestrians make up $22 \%$ of these fatalities [3]. According to the report, the situation is worst in low-income countries, where rates are more than double those in highincome countries. There is also a disproportionate number of deaths relative to these countries' level of motorization. Data reveals that $90 \%$ of road traffic deaths occur in low- and middle-income countries, yet these countries have just 54\% of the world's vehicles. Globally, an estimated 3\% of GDP is lost to road traffic deaths and injuries [3].

With a population of more than 1.37 billion, China has her traffic characteristics. First, the existing road traffic planning may not provide adequate safety for pedestrians in some cases. Hence, mixed traffic consisting of motorized vehicles and pedestrians is ubiquitous. Second, unsignalized crosswalks widely exist in small cities and suburbs.
Third, motorists are supposed to give way to pedestrians at unsignalized crosswalks, but these motorists do not always comply due to a general lack of safety consciousness $[4,5]$. Hence, the crossing behavior at unsignalized crosswalks is not as straightforward as it seems. It is of utmost importance to investigate the behavior of pedestrians and vehicles [1, 6-10] to inform policy-makers, pedestrians, and motorists better. Many microscopic simulation models, such as the social force model [11], cellular automaton model [12], and lattice gas model [13], have been used to investigate mixed traffic flows due to their simple, easy-to-calculate, and flexible characteristics. The motion characteristics of pedestrians and vehicles are accounted for in the models mentioned above. However, pedestrians and vehicles (drivers) are more than just moving objects. They are "rational people" and their cognitive and social processes need to be properly accounted for, though not easily achieved under these existing models.

Besides motion characteristics, behavioral characteristics like the cognitive, social, or even psychological behavior need to be properly accounted for in the model, to investigate the crossing behavior of pedestrians and vehicles thoroughly. Hence, modeling the pedestrian crossing behavior from the 
perspective of behavioral science is an essential process. Game theory is a subject of coordination of conflicts, suitable for studying crossing behavior. Although some studies have utilized game theory to investigate the crossing behavior [14-17], there are still many limitations that need to be overcome first. Most works assume that the decision-making process is a rational process, described by the prisoners' dilemma game. That is, they have a strategy that is either cooperative or defective. However, this does not necessarily agree with the real-life scenario. Secondly, some of these works also assume static games and fail to capture the dynamic processes and interactions between pedestrians and vehicles. Finally, some of these studies assume that the interactions of the individuals are based on simple behavioral rules, and all the individuals behave homogeneously. Most importantly, these studies fail to consider the physiological and psychological characteristics of individuals, as well as the traffic environment. Pedestrians' road crossing behavior may be influenced by many factors. These include physical environment (e.g. the number of vehicle lanes, types of street, with or without signals), road user variables (e.g. gender, age, education, income, the purpose of the trip, waiting time, attitude towards risk), and social factors (e.g. pedestrian group size at crosswalks).

It is worth noting that it has long been recognized that group behavior is not simply the sum of individual behaviors [18]. Intuitively, what we may not do as individuals; we may now do as part of a group. Human crowd dynamics has attracted the attention of researchers from many different fields. For instance, synergetics, fluid dynamics, molecular dynamics and cellular automata have all been applied to the study of social systems [19-23]. Statistical data indicates that up to $70 \%$ of people move in groups [24] and this motivates our current study to investigate the pedestrian groupcrossing behavior and its underlying dynamical mechanism. Moussaiid et al. [25] collected empirical data on the motion of pedestrian groups using video recordings in public areas and analyzed the organization of pedestrian social groups and their impact on the complex dynamics of crowd behavior. Zhou et al. [26, 27] examined the effects of conformity tendency on Chinese pedestrians' intentions to cross the road in potentially dangerous situations. The results reveal that pedestrians showed greater likelihood in crossing the road when other pedestrians were crossing the road. Rosenbloom [28] reviewed the road behavior of individual pedestrians and groups of pedestrians at these intersections. Their results indicate that $13.5 \%$ of the pedestrians arriving in the redlight phase crossed the street on a red light. Faria et al. [29] investigated social information use by pedestrians at a road crossing and assessed if pedestrians followed others by analyzing their starting positions and crossing orders. Their results show that on average a person was 1.5-2.5 times more likely to cross if their neighbor had started so. Xin et al. $[5,30]$ proposed a cellular automata model to study the characteristics of pedestrian-vehicle mixed traffic flows at the unsignalized crosswalk. Their study shows that pedestrians cross the crosswalk in groups, and the sizes of groups follow the power law distribution.

In reality, pedestrians and vehicles have to compete for limited road resources in a road-crossing scenario and this can be modeled as a "game" between pedestrians and vehicles. Through an in-depth analysis of the interference mechanism between groups of pedestrians and vehicles at the unsignalized intersections, a dirty faces game is used in this paper to analyze the crossing behavior of pedestrians and vehicles as "rational people". We first set up the multidimensional dirty faces game to analyze pedestrian groupcrossing behavior, where a dynamic process of modeling and simulation is then proposed. This paper fills an important gap because previous work [31] only considers one pedestrian and one vehicle at an intersection only. From our simulation results, the effect of the group size on crossing behavior and conflict risks is then analyzed.

\section{Multidimensional Dirty Faces Game of Pedestrian Group-Crossing Behavior}

The dirty faces game is well-studied and usually found in the literature on iterated reasoning. It plays a central role in most discussions of common knowledge [32, 33]. In an early description of the problem, Littlewood describes the following situation: "three ladies (A, B and $\mathrm{C}$ ) in a railway carriage all have dirty faces (but unable to observe their own faces) and are laughing at each other. As each lady notices that the others do not stop laughing, she infers that her own face must be dirty." Their thought process would be like this: "if I (in this case, A) am not laughable, then B will be arguing: if $\mathrm{I}$ (in this case, B) am not laughable, then $\mathrm{C}$ will have nothing to laugh at. Since B does not so argue, I (that is, A) must be laughable." Notice that, for A to realize this, A must think that $B$ is rational enough to draw an inference from $\mathrm{C}$, which is a subtle chain of reasoning. Readers can refer to Littlewood [34] for a detailed discussion on the dirty faces game. This logic can also be extended to an arbitrarily large number of dirty-faced ladies [34]. The dirty faces game relies on common knowledge about the fact that at least one face is dirty (which results from the public laughter) and on common knowledge of rationality [32].

In our study, we consider pedestrians and vehicle drivers as the two players of the game, where each player has two choices: strategy 1 is that the vehicle lets the pedestrian go first, and strategy 2 is that the vehicle goes first. The strategy chosen by the two players in areas of potential conflict will directly lead to the changes in intersection traffic conditions. By simple deduction, the following scenarios can be inferred.

(i) Both players choose strategy 1 (the vehicle lets the pedestrian go first); then the pedestrian decides to pass the intersection.

(ii) Both sides choose strategy 2 (the pedestrian lets the vehicle go first); then the vehicle decides to cross the intersection.

(iii) The pedestrian adopts strategy 1 and the vehicle adopts strategy 2 ; then the pedestrian and vehicle collide (this situation is very dangerous).

(iv) The pedestrian adopts strategy 2, while the vehicle adopts strategy 1 ; then both pedestrian and vehicle give way to the other (this situation is especially inefficient).

Through the above analysis, it is straightforward to see that both pedestrian and vehicle adopting strategy 1 or 
strategy 2 at the same time are the kind of situation that we would like to have. The key question is, how do we let pedestrian and vehicle adopt the same strategy simultaneously? The best approach is to make strategy 1 or strategy 2 becomes the common knowledge for both sides. Since both sides cannot communicate directly when they cross the road; therefore, they do not know which strategy his/her opponent will choose. At this moment, the choice between vehicle allowing the pedestrian to go first or the pedestrian allowing the vehicle to go first is not the common knowledge for both parties. Therefore, some factors that drive towards the formation of common knowledge must exist, and the signal lights in the intersection are exactly this driving factor. When the traffic light in the crosswalk turns green, the pedestrian sees that and adopts strategy 1; the vehicle also sees that and adopts strategy 1 . The pedestrian also knows that the vehicle will adopt strategy 1 , while the vehicle knows that the pedestrian will adopt strategy 1 . The pedestrian further knows that the vehicle is aware that the pedestrian will adopt strategy 1 ; the vehicle also knows that the pedestrian is aware that the vehicle will adopt strategy 1 as well. At this time, "the vehicle lets the pedestrian go first" becomes common knowledge for both sides. When the traffic light turns red, the analysis is the same as what we have just discussed. The only difference is that "the pedestrian lets the vehicle go first" becomes common knowledge for both sides at this time.

Since unsignalized crosswalks are ubiquitous in small cities in China, we now focus on the formation processes of common knowledge at these unsignalized crosswalks. Bayer et al. concluded that three elements are needed to form the common knowledge: (i) each player should generate a dominant strategy first, (ii) each player can assess and analyze the dominant strategy of the others, and (iii) each player can announce the dominant strategy of their own immediately. In order to obtain common knowledge at unsignalized intersections, pedestrian and vehicle usually produce and announce the dominant strategy of their own first according to the traffic condition and then assess his/her opponent's dominant strategy. The announcement and assessment cannot be explained in words and can only be shown in acceleration, deceleration, horn honking, or gesturing. The interactive announcement and assessment may be more than one round. Sometimes, several rounds of the game are needed to achieve common knowledge, and common knowledge may not even be fully achieved in some instances.

The "Chinese style road crossing" refers to the behavior of Chinese pedestrians crossing an intersection, not by following the traffic signals but whether there is a sufficient number of pedestrians who feel safe to cross $[35,36]$. Pedestrians running the red light are a common phenomenon that can be observed in many countries [37, 38]. Even though traffic lights exist, there are still many reasons that will render vehicle drivers and pedestrians to disobey them and the situation at unsignalized crosswalks will be even worse. A possible explanation from the perspective of the multidimensional dirty faces game is as follows. Assume that the vehicle driver (for continuous vehicle flows, we only consider the heading car) and the waiting pedestrians are the players of the game. Only when the driver and all waiting pedestrians form the common knowledge of "the pedestrian lets the vehicle go first," then the vehicle passes through the intersection. In fact, the driver may not choose all pedestrians as competitors, where only representative pedestrians are selected as competitors in the game. In practice, the representative pedestrian can be the one who stands in front of the crowd or the one who demonstrates an intention to cross (i.e., stretch his head and look out or suddenly accelerates). If the driver and representative pedestrians form common knowledge, the other pedestrians will recognize the strategy under conformity pressure. Thus, the dimension of the dirty faces game is reduced. The solution to the simplified game can then be used as the solution to the multidimensional dirty faces game. This works in most cases. However, there are some special cases that need to be considered separately. For instance, the waiting time of pedestrians may be too long and some of the pedestrians are not patient enough; the gap between two adjacent vehicles is large enough; or someone from the pedestrian group (usually the one not selected as a representative pedestrian) changes the strategy unilaterally and takes the lead to cross. If the driver is forced to accept the strategy, the other pedestrians change their strategy and begin to cross. In this way, the common knowledge of "the pedestrian lets the vehicle go" changes to one of "the vehicle lets the pedestrian go." Based on the multidimensional dirty faces game, we now propose a systematic way to analyze crossing behavior of pedestrians and vehicles as follows.

Step 1. For a pedestrian group which consists of $N$ pedestrians waiting to cross, the probability that the $i$ th pedestrian selects "the vehicle driver gives priority to the pedestrian" as the dominant strategy is

$$
P_{p}^{i}=\left\{\begin{array}{ll}
\frac{T_{p i}^{c}-T_{p i}^{p i}}{T_{p i}^{c}} & T_{p i}^{c}>T_{p i}^{p i} \\
0 & T_{p i}^{c} \leq T_{p i}^{p i}
\end{array} \quad(i=1,2, \ldots, N),\right.
$$

where $T_{p i}^{c}$ represents the passing time of the vehicle estimated by the $i$ th pedestrian on the basis of traffic parameters and $T_{p i}^{p i}$ represents the passing time of the $i$ th pedestrian estimated by himself on the basis of traffic parameters. Assume that the estimated passing time satisfies the normal distribution, where the average value of the distribution is equal to the theoretical passing time, and the standard deviations $\sigma$ and $o$ reflect the estimation accuracy of the pedestrian and driver, respectively. Let $\mu_{i}$ be the theoretical passing time of the $i$ th pedestrian, $\mu_{i}=H / v_{p i}, H$ be the road width, $v_{p i}$ be the walking speed of the $i$ th pedestrian, $u$ be the theoretical passing time of the vehicle, where $u=L / v_{c}, L$ be the distance between the vehicle and the point of intersection, and $v_{c}$ be the vehicle speed. In the proposed simulation, $T_{p i}^{c}$ and $T_{p i}^{p i}$ are random numbers satisfying the normal distributions $N\left(u, \sigma_{i}\right)$ and $N\left(u_{i}, \sigma_{i}^{*}\right)$, respectively, where $\sigma_{i}=k_{i} u, \sigma_{i}^{*}=k_{i}^{*} \mu_{i}$, and $k_{i}$ and $k_{i}^{*}$ reflect the pedestrian's estimation accuracy of the passing times of himself and the vehicle, respectively. The probability that the $i$ th pedestrian selects "the pedestrian gives priority to the vehicle" as the dominant strategy is $1-P_{p}^{i}$. 
The probability that the vehicle driver selects "the pedestrian gives priority to vehicle" as the dominant strategy is given by $P_{c}$ and its derivation can be found in Appendix A.

Step 2. Generate $N+1$ random numbers: $\gamma_{i} \in[0,1],(i=$ $1,2, \ldots, N+1)$. Then, condition (2) below can be used to assess whether the pedestrians and driver can achieve common knowledge. If common knowledge can be achieved, the pedestrians or driver will pass through the intersection under the zero-step mode, and the computations can be completed. Otherwise, go to the one-step mode.

For any $\gamma_{i} \leq P_{p}^{i}(i \in\{1,2, \ldots, N\}), \gamma_{N+1}>P_{c}$, both sides select strategy 1 as the dominant strategy, common knowledge is achieved, the pedestrians pass through the intersection, For all $\gamma_{i}>P_{p}^{i}(i \in\{1,2, \ldots, N\}), \gamma_{N+1} \leq P_{c}$, both sides select strategy 2 as the dominant strategy, common knowledge is achieved, the vehicles pass through the intersection, For all $\gamma_{i}>P_{p}^{i}(i \in\{1,2, \ldots, N\}), \gamma_{N+1}>P_{c}$, both sides have no willingness to cross, common knowledge is not achieved, they give the way to each other, For any $\gamma_{i} \leq P_{p}^{i}(i \in\{1,2, \ldots, N\}), \gamma_{N+1} \leq P_{c}$, at least one pedestrian intends to cross and the vehicle intends to cross, common knowledge is not achieved, both sides are willing to cross.

Step 3. When both pedestrians and driver enter the onestep mode, they further announce the dominant strategy of their own via acceleration or deceleration, and they may also recognize the dominant strategy of the other. As such, we define two parameters $\eta_{p}^{i}$ and $\eta_{c}$ that describe the possibility of acceleration or deceleration for pedestrians and vehicles, respectively. According to condition (1) and (2) above, the person with a higher probability to produce a dominant strategy, will have a shorter estimated passing times. It is reasonable to assume that this same person will have a higher probability of acceleration when entering the one-step mode. This can be represented in the following form:

$$
\eta_{p}^{i}=\frac{P_{p}^{i}}{P_{p}^{i}+P_{c}}
$$

$$
\begin{aligned}
& \eta_{c}^{i}=\frac{P_{c}}{P_{p}^{i}+P_{c}}, \\
& \eta_{c}=\min _{i=1}^{N} \eta_{c^{\prime}}^{i}
\end{aligned}
$$

Now, we generate $N+1$ random numbers, $\lambda_{i} \in[0,1], \quad(i=$ $1,2, \ldots, N+1)$. Then, condition (4) below can be used to decide whether the pedestrians and driver can achieve common knowledge. If common knowledge can be achieved, the pedestrians or driver will pass through the intersection under the one-step mode, and the computations are completed. Otherwise, go to the two-step mode.

For any $\lambda_{i} \leq \eta_{p}^{i}(i \in\{1,2, \ldots, N\}), \lambda_{N+1}>\eta_{c}$, at least one pedestrian begins to accelerate and the vehicle begins to decelerate, common knowledge is achieved, the pedestrians pass through the intersection,

For all $\lambda_{i}>\eta_{p}^{i}(i \in\{1,2, \ldots, N\}), \lambda_{N+1} \leq \eta_{c}$, all pedestrians begin to decelerate and the vehicle begins to accelerate, common knowledge is achieved, vehicles pass through the intersection,

For all $\lambda_{i}>\eta_{p}^{i}(i \in\{1,2, \ldots, N\}), \lambda_{N+1}>\eta_{c}$, all pedestrians and the vehicle begin to decelerate, common knowledge is not achieved, they give the way to each other,

For any $\lambda_{i} \leq \eta_{p}^{i}(i \in\{1,2, \ldots, N\}), \lambda_{N+1} \leq \eta_{c}$, at least one pedestrian begins to accelerate and the vehicle begins to accelerate, common knowledge is not achieved, both sides are willing to cross.

Step 4. Generate $N+1$ random numbers, $\delta_{i} \in[0,1],(i=1$, $2, \ldots, N+1$ ). We define $\eta_{p}^{i 2}$ and $\eta_{c}^{2}$ (both pedestrian and driver become more cautious) as the possibility of acceleration by the pedestrians and vehicle, respectively, at this stage. Then, condition (5) below can be used to decide whether the pedestrians and driver can achieve common knowledge. 
If common knowledge can be achieved, the pedestrian or driver will pass through the intersection under the twostep mode, and the computations are completed. Otherwise, both sides fail to cross the intersection normally and this leads to the failure mode whereby the dirty faces game is terminated. Failure mode corresponds to the stagnation or collision between pedestrians and vehicle in reality.

For any $\delta_{i} \leq \eta_{p}^{i 2}(i \in\{1,2, \ldots, N\}), \delta_{N+1}>\eta_{c}^{2}$, at least one pedestrian begins to accelerate and the vehicle begins to decelerate, common knowledge is achieved, the pedestrians pass through the intersection,

For all $\delta_{i}>\eta_{p}^{i 2}(i \in\{1,2, \ldots, N\}), \delta_{N+1} \leq \eta_{c}^{2}$, all pedestrians begin to decelerate and the vehicle begins to accelerate, common knowledge is achieved, the vehicle passes through

the intersection,

For all $\delta_{i}>\eta_{p}^{i 2}(i \in\{1,2, \ldots, N\}), \delta_{N+1}>\eta_{c}^{2}$, all pedestrians and the vehicle begin to decelerate, common knowledge is not achieved, stagnation of the pedestrians and vehicle $\longrightarrow$ the failure mode, For any $\delta_{i} \leq \eta_{p}^{i 2}(i \in\{1,2, \ldots, N\}), \delta_{N+1} \leq \eta_{c}^{2}$, at least one pedestrian begins to accelerate and the vehicle begins to accelerate, common knowledge is not achieved, collision between the pedestrians and vehicle $\longrightarrow$ the failure mode.

Figure 1 presents a schematic diagram to illustrate the dynamic process of pedestrian group-crossing behavior. Our simulation results predict that, with increasing $N$, the probability of the pedestrian group and vehicle forming the common knowledge of "the pedestrian gives priority to the vehicle" and they give way to each other decreases, while the probability of the pedestrian group and vehicle forming the common knowledge of "the vehicle driver gives priority to the pedestrian" and both sides cross simultaneously increases. Considering that only the representative pedestrians are selected as competitors in the game, we take the representative pedestrians to replace all $N$ pedestrians. Thus, the dimension of the dirty faces game is reduced. The Monte-Carlo simulation approach is then applied to mimic a real roadcrossing scenario by relying on repeated random sampling to obtain numerical results. The pseudocode implementation of the Monte-Carlo simulation is given in Appendix B.

\section{Simulation Results and Discussion}

3.1. Simulation Analysis in Consideration of the Heterogeneity of Waiting Time. Due to differences in arrival times and traffic conditions, different pedestrians may experience different waiting times. When the waiting time increases, pedestrians become impatient and try to cross the street impetuously $[39,40]$. Therefore, the heterogeneity of waiting time may affect the crossing behavior of pedestrian groups. We assume that different pedestrians in the group experience different waiting times, and the waiting times satisfy the uniform distribution $T \sim U(0,70)$. In order to quantify the influence of the waiting time on the crossing behavior of pedestrians, we adopt the method proposed by Wang et al. [31]. We introduce the influence coefficient of the waiting time $\xi(t)$, which reflects the amplification effect of the crossing probability due to an increase in the waiting time.
Accordingly, the probability that the $i$ th pedestrian selects "the vehicle gives priority to the pedestrian" as the dominant strategy is $\min \left(\xi\left(t_{i}\right) P_{p}, 1\right)$

$$
\xi(t)=\frac{1}{1+e^{-0.2(t-35)}}+1 .
$$

Other parameters remain unchanged. Simulations were performed to investigate the effect of the waiting time on the crossing behavior of pedestrians. In the simulation model, it is assumed that each pedestrian in the group has the same theoretical passing time (i.e., they have the same walking speed) and estimation accuracy, where the estimated passing times satisfy the same normal distribution.

The simulation was performed under different combinations of $\mu$ (the theoretical passing time of a pedestrian) and $u$ (the theoretical passing time of the vehicle). The number of samples is $1,000,000$. Figure 2 shows the effect of the group size on the conflict probability, where the number of pedestrians is $1,2,3,5,10$, and 20, respectively. The conflict probability here is defined as the ratio of the sample that causes a conflict in the failure mode to the sample size. The simulation results predict that, with an increase in the group size, the size of the region of the parameter space that may cause a conflict decreases. At the same time, the conflict probability also decreases.

In order to further explore the effect of the group size on the decision mechanism of crossing behavior, we tracked the number of different crossing results for two combinations of $\mu$ and $u$ (case 1: the theoretical passing time of pedestrians $\mu=4 \mathrm{~s}$, the theoretical passing time of the vehicle $u=4 \mathrm{~s}$; case 2: $\mu=4 \mathrm{~s}, u=3 \mathrm{~s}$ ). The Monte-Carlo simulation results are given in Tables 1 and 2 for illustrative purposes.

Table 1 shows that when the theoretical passing times of both pedestrians and vehicle are $4 \mathrm{~s}$, the passing probability 


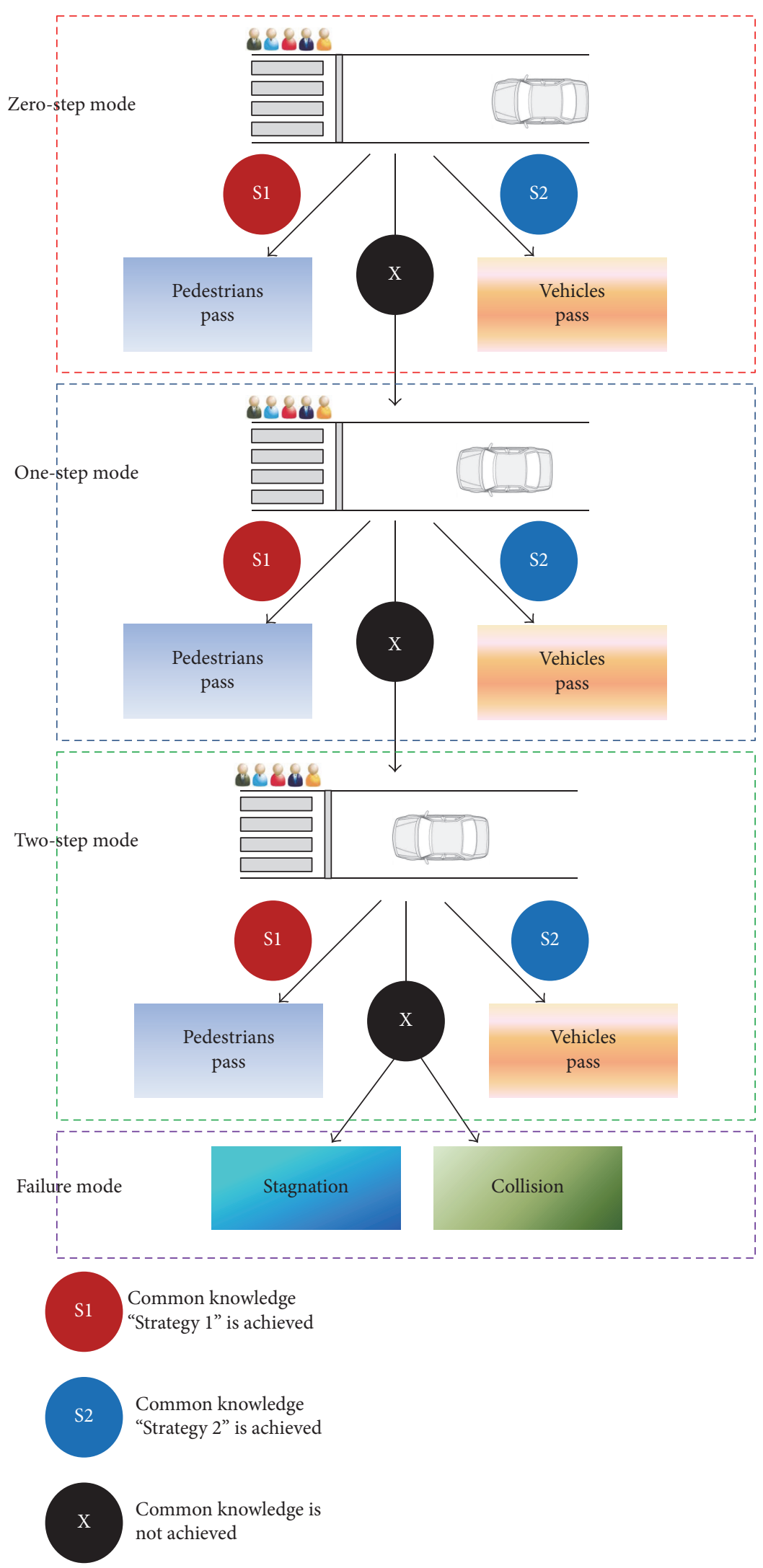

FIGURE 1: Schematic diagram to illustrate the multidimensional dirty faces game of pedestrian group-crossing behavior. 
TABLE 1: Number (out of the sample of 1 million) of pedestrians/vehicles passing through the intersection under different modes $(\mu=4 \mathrm{~s}$, $u=4 \mathrm{~s})$.

\begin{tabular}{lcccccccc}
\hline \multirow{2}{*}{$\begin{array}{l}\text { Number of } \\
\text { pedestrians }\end{array}$} & \multicolumn{2}{c}{ Zero-step mode } & \multicolumn{2}{c}{ One-step mode } & \multicolumn{2}{c}{ Two-step mode } & \multicolumn{2}{c}{ Failure mode } \\
& $\begin{array}{c}\text { Pedestrians } \\
\text { pass }\end{array}$ & $\begin{array}{c}\text { Vehicles } \\
\text { pass }\end{array}$ & $\begin{array}{c}\text { Pedestrians } \\
\text { pass }\end{array}$ & $\begin{array}{c}\text { Vehicles } \\
\text { pass }\end{array}$ & $\begin{array}{c}\text { Pedestrians } \\
\text { pass }\end{array}$ & $\begin{array}{c}\text { Vehicles } \\
\text { pass }\end{array}$ & Stagnation & Collision \\
\hline 1 & 104861 & 66740 & 320947 & 319672 & 40807 & 36828 & 99449 & 10696 \\
2 & 205403 & 32584 & 464945 & 139396 & 45129 & 23831 & 79336 & 9376 \\
3 & 293856 & 19750 & 510476 & 65228 & 36746 & 13827 & 53544 & 6573 \\
5 & 445324 & 9261 & 477980 & 16943 & 19625 & 4588 & 23455 & 2824 \\
10 & 691310 & 2405 & 291102 & 1362 & 4914 & 534 & 7907 & 466 \\
20 & 906194 & 322 & 91674 & 52 & 1044 & 26 & 672 & 16 \\
\hline
\end{tabular}

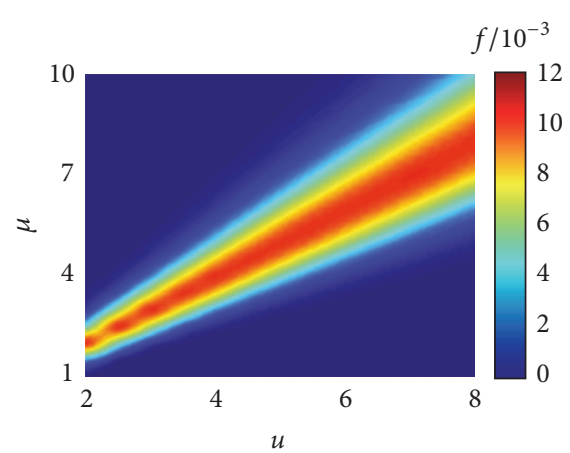

(a)

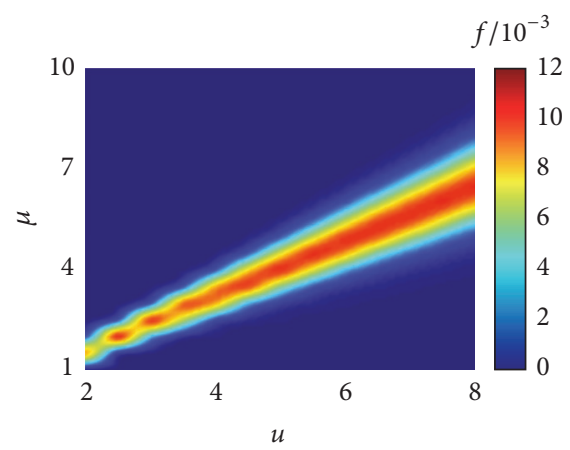

(d)

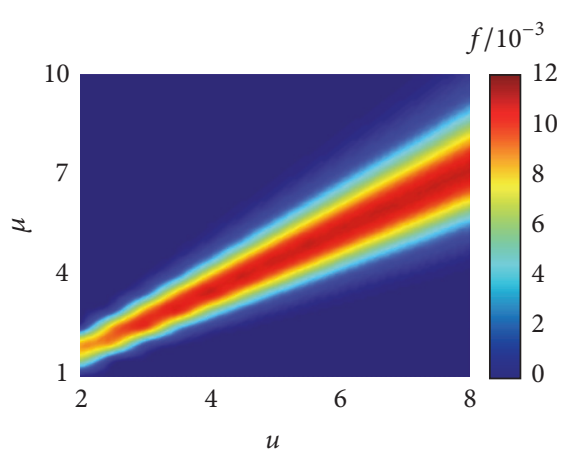

(b)

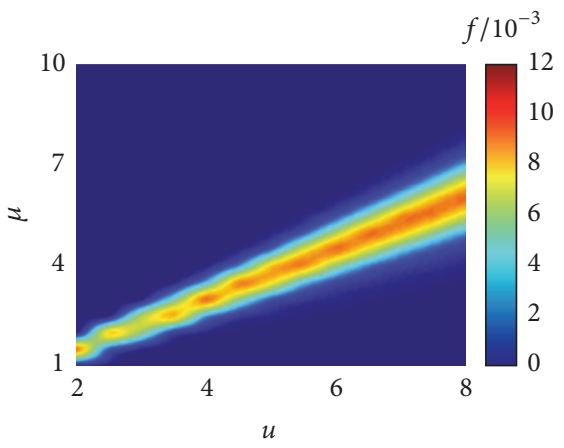

(e)

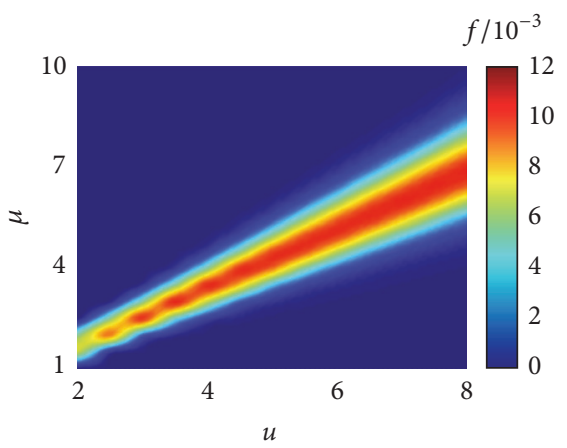

(c)

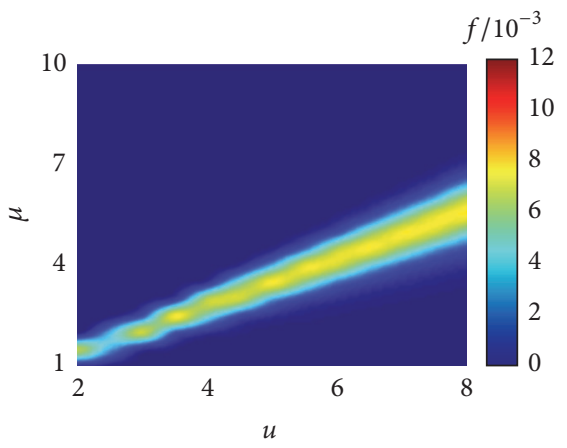

(f)

Figure 2: The effect of the group size on the conflict probability in consideration of the heterogeneity of the waiting time (the number of pedestrians is $1,2,3,5,10$, and 20 , from (a) to (f) graph). $k_{i}=k_{i}^{*}=0.15, l=l^{*}=0.15$.

of pedestrians is higher than that of the vehicle in all modes. This is because the probability that pedestrians try to cross the street increases with an increase in the waiting time. This phenomenon becomes more pronounced with an increase in the group size. Specifically, when the group size is 5 , nearly $95 \%$ of pedestrians cross the intersection successfully before the failure mode. Moreover, the conflict probability decreases with an increase in the group size. This is because the probability that the driver selects "the pedestrian gives priority to the vehicle" as the dominant strategy decreases; this makes more pedestrians cross the intersection successfully before the failure mode. The above analysis appears to support the notion of "the more, the safer" behavior of pedestrians and can elucidate the "Chinese style road crossing" phenomenon.

As shown in Table 2, when the theoretical passing time of pedestrians is $4 \mathrm{~s}$ and the theoretical passing time of the vehicle is $3 \mathrm{~s}$; the vehicle has the advantage in crossing without considering other factors. When there is only one pedestrian, the passing probability of the vehicle is much larger than that of pedestrians under all modes. It is worth noting that the probability that the vehicle and pedestrians complete crossing under zero-step mode is not high because the advantage of the passing time is not immediately obvious for the vehicle. However, under the one-step mode, they obtain common knowledge with a larger probability and complete the crossing. The probability that the pedestrians cross the intersection successfully increases with an increase in the group size. When the group size is 10 , the proportions of pedestrians and vehicles passing through the intersection are approximately equal. When the group size is 20 , the ratio of pedestrians passing through the intersection is more than $60 \%$. This is because, with an increase in the group size, the probability that the driver selects "the pedestrian gives 
TABLE 2: Number (out of the sample of 1 million) of pedestrians/vehicles passing through the intersection under different modes $(\mu=4 \mathrm{~s}$, $u=3 \mathrm{~s})$.

\begin{tabular}{|c|c|c|c|c|c|c|c|c|}
\hline \multirow{2}{*}{$\begin{array}{l}\text { Number of } \\
\text { pedestrians }\end{array}$} & \multicolumn{2}{|c|}{ Zero-step mode } & \multicolumn{2}{|c|}{ One-step mode } & \multicolumn{2}{|c|}{ Two-step mode } & \multicolumn{2}{|c|}{ Failure mode } \\
\hline & $\begin{array}{c}\text { Pedestrians } \\
\text { pass }\end{array}$ & $\begin{array}{l}\text { Vehicles } \\
\text { pass }\end{array}$ & $\begin{array}{l}\text { Pedestrians } \\
\text { pass }\end{array}$ & $\begin{array}{l}\text { Vehicles } \\
\text { pass }\end{array}$ & $\begin{array}{l}\text { Pedestrians } \\
\text { pass }\end{array}$ & $\begin{array}{l}\text { Vehicles } \\
\text { pass }\end{array}$ & Stagnation & Collision \\
\hline 1 & 10360 & 239795 & 37148 & 652438 & 10692 & 14407 & 31766 & 3394 \\
\hline 2 & 21910 & 179492 & 74377 & 617891 & 19958 & 23732 & 56776 & 5864 \\
\hline 3 & 47495 & 166282 & 94645 & 569087 & 23235 & 28254 & 65019 & 5983 \\
\hline 5 & 80325 & 134685 & 149506 & 472117 & 32868 & 34685 & 88424 & 7390 \\
\hline 10 & 141327 & 85536 & 221891 & 265069 & 39024 & 32741 & 200580 & 13832 \\
\hline 20 & 268657 & 54876 & 303924 & 115707 & 38807 & 23802 & 183627 & 10600 \\
\hline
\end{tabular}

priority to the vehicle" as the dominant strategy decreases. At the same time, the probability that the pedestrians try to cross the street impetuously increases, and this results in a higher probability that they obtain the common knowledge "the pedestrians pass first." Besides, the probability that both sides enter the failure mode increases with an increase in the group size; especially, the probability that the pedestrians and vehicle yield to each other increases. This is because the vehicle has the advantage in crossing times and pedestrians have the advantage in numbers, and which one occupies the dominant status is not clear at all. When both sides are very hesitant, if one side happens to be radical, the probability of a collision greatly increases.

3.2. Simulation Analysis in Consideration of the Heterogeneity of Walking Speed. In Section 3.1, we analyzed the situation in which each pedestrian in the group has the same theoretical passing time. However, in real life, different people have different walking speeds due to their differences in ages, genders, physical conditions, and travel purposes. Statistical data show that the walking speeds of pedestrians satisfy the normal distribution [41]. Based on statistical data, we can assume that the average walking speed is $1.3 \mathrm{~m} / \mathrm{s}$ and the standard deviation is 0.15 times the average walking speed, that is, $V_{p} \sim N(1.3,0.195)$. Simulations were performed with the road width as $H=5 \mathrm{~m}$. Thus, the average theoretical crossing time of a pedestrian is $3.85 \mathrm{~s}$.

Figure 3 shows the crossing probability for pedestrians for different group sizes (the theoretical passing time of vehicles is set to $4 \mathrm{~s}$ ). Since the average theoretical passing time of pedestrians is approximately equal to the theoretical passing time of the vehicle, we can see from the figure that when there is only one pedestrian, the crossing probability for pedestrians is about $50 \%$. This demonstrates that the pedestrian and vehicle have equal opportunity to cross. With an increase in the group size, the crossing probability for pedestrians increases as well. When there are ten pedestrians, the crossing probability for pedestrians approaches $100 \%$.

Figure 4 shows the crossing probability for pedestrians for different theoretical passing times of vehicles (the number of pedestrians is set to 20). When the theoretical passing time of vehicles is $3 \mathrm{~s}$, the crossing probability for pedestrians is nearly $100 \%$. Though the vehicle benefits from a better theoretical passing time $(3 \mathrm{~s}<3.85 \mathrm{~s})$, the probability that the theoretical passing time of a specific pedestrian is less

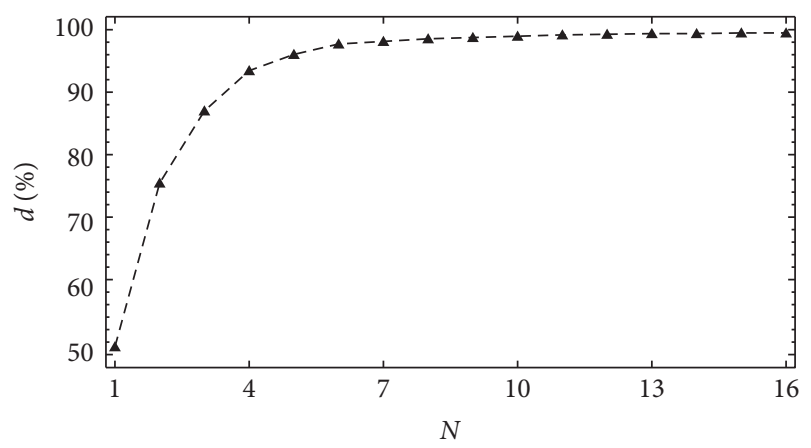

FIgURE 3: The crossing probability for pedestrians for different group sizes ( $d$ is the ratio of the sample number of pedestrians crossing to the total sample size; the theoretical time of vehicles is $u=L / v_{c}=4 \mathrm{~s}$; the total sample size is $1,000,000$ ).

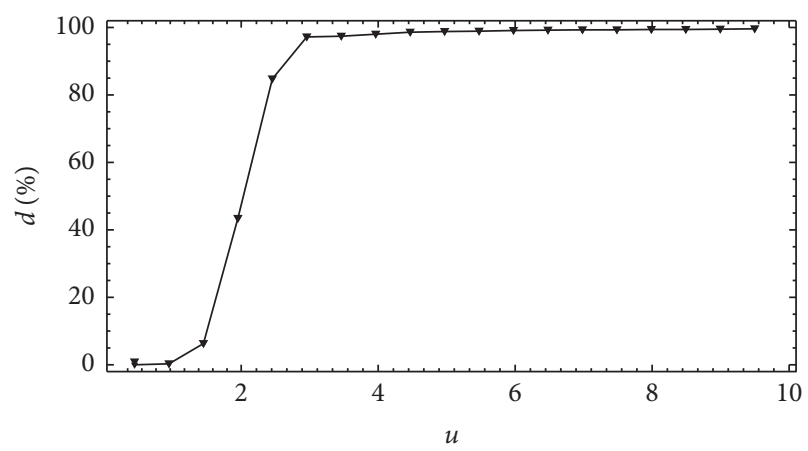

FIgURE 4: The crossing probability for pedestrians for different theoretical times of vehicles (the number of pedestrians is 20 ; the total sample size is $1,000,000$ ).

than $3 \mathrm{~s}$ increases with the increase in the group size. When a specific pedestrian takes the lead to cross and the other pedestrians follow suit, hence, the advantage in having a higher numbers of pedestrians induces the vehicle to give way to the pedestrians instead.

Figure 5 shows the collision probability, $f$ for different theoretical passing times of vehicles in consideration of the group size. The results show that, with an increase in the group size, the size of the parameter space that may cause a conflict decreases. This is because the probability that a specific pedestrian has a higher walking speed within the group increases with an increase in the group size, and 


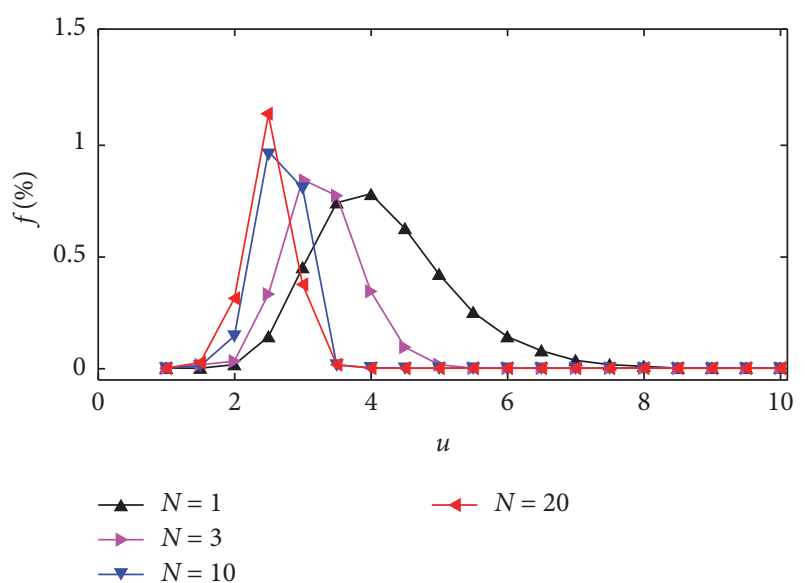

FIGURE 5: The collision probability $f$ for different theoretical times of vehicles in consideration of the group size.

more pedestrians cross the intersection successfully before the failure mode. At the same time, the peak of the collision probability becomes larger with an increase in the group size. Through the analysis of the theoretical passing times of vehicles corresponding to the peak of the collision probability, we can deduce that the theoretical passing time of the vehicle is smaller than that of the average pedestrian. This means that the probability that the driver selects "the pedestrian gives priority to the vehicle" as the dominant strategy is relatively high. At the same time, the advantage of having a higher numbers of pedestrians leads them to change their former strategy and to cross the intersection. Thus, the probability of a collision increases. Group-crossing risks can be interpreted as a "gamble": it saves time for pedestrians and will also lead to a higher probability of a collision.

\section{Conclusion}

Using the multidimensional dirty faces game, coupled by Monte-Carlo simulations, we have proposed an original and novel method to analyze the crossing behavior of pedestrians group-behavior and vehicles as "rational people." Our model is able to capture the multistage dynamic interactions between pedestrians and vehicles.

By considering the heterogeneity of waiting times, our results show that, with an increase in the group size, the size of the parameter space that may cause a conflict decreases, while the conflict probability also decreases. When the theoretical passing times of both pedestrians and vehicle are equal, the crossing probability of pedestrians is higher than that of the vehicle in all modes. This phenomenon becomes more pronounced with an increase in the group size. When the theoretical passing time of the vehicle is smaller than the theoretical passing times of pedestrians, the probability that the pedestrians cross the intersection increases, with an increase in the group size. At the same time, the probability that both sides enter the failure mode or encounter a collision increases. Considering the heterogeneity of walking speeds and an increase in the group size, the crossing probability for pedestrians becomes larger, and the size of the parameter space that may cause a conflict decreases. However, the probability that both sides enter the failure mode or have a collision increases. Through an in-depth analysis of the theoretical passing time of the vehicle corresponding to the peak of the collision probability, we observe that the theoretical passing time of the vehicle is smaller than that of the average pedestrian.

Our findings have provided a theoretical framework to advance understanding of the illegal pedestrian groupcrossing behavior and elucidate the dynamics involved. Future work includes the use of empirical data to validate the proposed model. The passing ratios of pedestrians/vehicles crossing and the ratios of stagnation and collision can be obtained in a well-designed observational study. However, since this is a multistage dynamic model, it is not easy to fully capture the interaction process (i.e., the acceleration probabilities in each mode) from an empirical study. To this end, more advanced technologies or restricting the experiments in a laboratory setting should be explored. This paper mainly focuses on analyzing the crossing behavior at unsignalized intersections with the assumption that every pedestrian has the intention to cross without considering the traffic signals. However, this is not always the case at signalized intersections. Future work will involve overcoming the limitations mentioned above. These result findings will also be useful to policy-makers for intersection design, informing pedestrian road safety, and minimizing pedestrian and vehicle collision. Traffic rules define that motor vehicle should slow down and let the pedestrians cross first. However, vehicle drivers do not always comply due to the lack of safety consciousness, thereby increasing conflict risk. The government may want to look into increasing the penalty and ways to standardize drivers' driving behavior. Pedestrian islands can also be used to help pedestrians cross roads safely, where pedestrians can stop before finishing crossing wide, multilane busy roads.

\section{Appendix}

\section{A. Calculation of the Probability That the Vehicle Driver Selects "the Pedestrian Giving Priority to Vehicle" as the Dominant Strategy}

The probability that the vehicle driver selects "the pedestrian gives priority to vehicle" as the dominant strategy is

$$
P_{c}=\min _{i=1}^{N} P_{c}^{p i}
$$

where

$$
P_{c}^{p i}=\left\{\begin{array}{ll}
\frac{T_{c}^{p i}-T_{c}^{c}}{T_{c}^{p i}} & T_{c}^{p i}>T_{c}^{c} \\
0 & T_{c}^{p i} \leq T_{c}^{c}
\end{array} \quad(i=1,2, \ldots, N)\right.
$$

where $T_{c}^{p i}$ represents the passing time of the $i$ th pedestrian estimated by the vehicle on the basis of traffic parameters, and $T_{c}^{c}$ represents the passing time of the vehicle estimated by the 


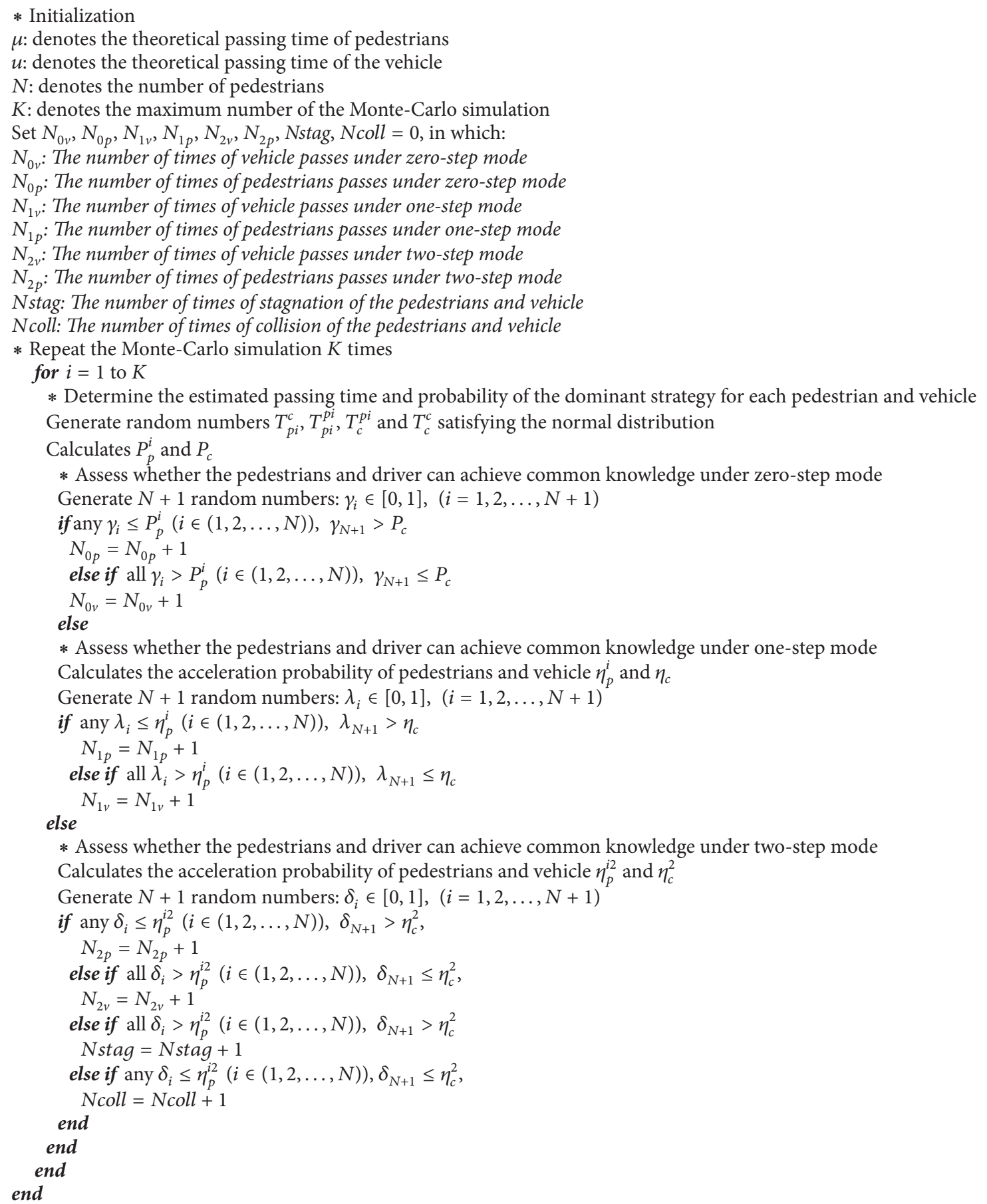

Pseudocode 1: Pseudocode implementation of the Monte-Carlo simulation.

driver himself on the basis of traffic parameters. In the same way, $T_{c}^{p i}$ and $T_{c}^{c}$ are random numbers satisfying the normal distribution $N\left(\mu_{i}, o_{i}\right)$ and $N\left(u, o^{*}\right)$, respectively, where $o_{i}=$ $l \mu_{i}, o^{*}=l^{*} u$, and $l$ and $l^{*}$ reflect the vehicle driver's estimation accuracy of the passing time of the pedestrian and himself, respectively. The probability that the vehicle driver selects "the vehicle driver gives priority to the pedestrian" as the dominant strategy is $1-P_{c}$.

\section{B. Pseudocode Implementation of the Monte-Carlo Simulation}

See Pseudocode 1.

\section{Conflicts of Interest}

The authors declare that they have no conflicts of interest. 


\section{Acknowledgments}

This project was supported by the National Natural Science Foundation of China (Grant no. 61375068), Ministry of Education, Humanities and Social Sciences research projects (13YJAZH106 and 15YJCZH210), Anhui Provincial Natural Science Foundation (1708085MF164), and Talent Project for Higher Education Promotion Program of Anhui Province.

\section{References}

[1] J. Yang, W. Deng, J. Wang, Q. Li, and Z. Wang, "Modeling pedestrians' road crossing behavior in traffic system microsimulation in China," Transportation Research Part A: Policy and Practice, vol. 40, no. 3, pp. 280-290, 2006.

[2] G. Asaithambi and M. O. Kuttan, "Pedestrian road crossing behavior under mixed traffic conditions: a comparative study of an intersection before and after implementing control measures," Transportation in Developing Economies, vol. 2, no. 2, pp. $1-12,2016$.

[3] World Health Organization (2015). Global ststus report on road safety. http://www.who.int/violence_injury_prevention/road_ safety_status/2015/en/.

[4] S. Turner, K. Fitzpatrick, M. Brewer, and E. S. Park, "Motorist yielding to pedestrians at unsignalized intersections findings from a national study on improving pedestrian safety," Transportation Research Record, no. 1982, pp. 1-12, 2006.

[5] X. Xin, N. Jia, S. Ma, and J. Jian, "Power laws in the cluster sizes of group-crossing pedestrians: empirical evidence and modelling of causes," Journal of Statistical Mechanics: Theory and Experiment, vol. 11, 11029 pages, 2015.

[6] M. M. Hamed, "Analysis of pedestrians' behavior at pedestrian crossings," Safety Science, vol. 38, no. 1, pp. 63-82, 2001.

[7] R. Lobjois and V. Cavallo, "Age-related differences in streetcrossing decisions: The effects of vehicle speed and time constraints on gap selection in an estimation task," Accident Analysis \& Prevention, vol. 39, no. 5, pp. 934-943, 2007.

[8] A. Dommes, V. Cavallo, and J. Oxley, "Functional declines as predictors of risky street-crossing decisions in older pedestrians," Accident Analysis \& Prevention, vol. 59, pp. 135-143, 2013.

[9] A. Dommes, V. Cavallo, J.-B. Dubuisson, I. Tournier, and F. Vienne, "Crossing a two-way street: Comparison of young and old pedestrians," Journal of Safety Research, vol. 50, pp. 27-34, 2014.

[10] P. Onelcin and Y. Alver, "Illegal crossing behavior of pedestrians at signalized intersections: Factors affecting the gap acceptance," Transportation Research Part F: Traffic Psychology and Behaviour, vol. 31, pp. 124-132, 2015.

[11] D. Helbing, "A mathematical model for the behavior of pedestrians," Behavioural Science, vol. 36, no. 4, pp. 298-310, 1991.

[12] V. J. Blue and J. L. Adler, "Cellular automata microsimulation for modeling bi-directional pedestrian walkways," Transportation Research Part B: Methodological, vol. 35, no. 3, pp. 293-312, 2001.

[13] D. Helbing, M. Isobe, T. Nagatani, and K. Takimoto, "Lattice gas simulation of experimentally studied evacuation dynamics," Physical Review E, vol. 67, pp. 490-511, 2003.

[14] M. Perc, "Premature seizure of traffic flow due to the introduction of evolutionary games," New Journal of Physics, vol. 9, article A3, 2007.

[15] W. Zhang, W. Zhang, and W. Chen, "Dilemma game in a cellular automaton model with a non-signalized intersection," The European Physical Journal B, vol. 85, no. 2, pp. 1-8, 2012.
[16] G. Abramson, V. Semeshenko, and J. R. Iglesias, "Cooperation and defection at the crossroads," PLoS ONE, vol. 8, no. 4, article e61876, 2013.

[17] G. Paissan and G. Abramson, "Imitation dynamics in a game of traffic," The European Physical Journal B, vol. 86, no. 4, pp. 1-6, 2013.

[18] J. S. Koopman and J. W. Lynch, "Individual causal models and population system models in epidemiology," American Journal of Public Health, vol. 89, no. 8, pp. 1170-1174, 1999.

[19] D. Brockmann, L. Hufnagel, and T. Geisel, "The scaling laws of human travel," Nature, vol. 439, no. 7075, pp. 462-465, 2006.

[20] D. Helbing, P. Molnár, I. J. Farkas, and K. Bolay, "Self-organizing pedestrian movement," Environment and Planning B: Planning and Design, vol. 28, no. 3, pp. 361-383, 2001.

[21] R. L. Hughes, "The flow of human crowds," Annual Review of Fluid Mechanics, vol. 35, no. 1, pp. 169-182, 2003.

[22] R. L. Hughes, "A continuum theory for the flow of pedestrians," Transportation Research Part B: Methodological, vol. 36, no. 6, pp. 507-535, 2002.

[23] Y.-Q. Jiang, P. Zhang, S. C. Wong, and R.-X. Liu, "A higher-order macroscopic model for pedestrian flows," Physica A: Statistical Mechanics and its Applications, vol. 389, no. 21, pp. 4623-4635, 2010.

[24] N. Guo, J. Ding, X. Ling, I. Takashi, and Q. Shi, “The walking behavior of pedestrian groups in the merchandise streets," in Proceedings of the 12th COTA International Conference of Transportation Professionals: Multimodal Transportation Systems Convenient, Safe, Cost-Effective, Efficient, CICTP 2012, pp. 649659, August 2012.

[25] M. Moussaïd, N. Perozo, S. Garnier, D. Helbing, and G. Theraulaz, "The walking behaviour of pedestrian social groups and its impact on crowd dynamics," PLoS ONE, vol. 5, no. 4, article e10047, 2010.

[26] R. Zhou, W. J. Horrey, and R. Yu, "The effect of conformity tendency on pedestrians' road-crossing intentions in China: an application of the theory of planned behavior," Accident Analysis \& Prevention, vol. 41, no. 3, pp. 491-497, 2009.

[27] R. Zhou and W. J. Horrey, "Predicting adolescent pedestrians' behavioral intentions to follow the masses in risky crossing situations," Transportation Research Part F: Traffic Psychology and Behaviour, vol. 13, no. 3, pp. 153-163, 2010.

[28] T. Rosenbloom, "Crossing at a red light: behaviour of individuals and groups," Transportation Research Part F: Traffic Psychology and Behaviour, vol. 12, no. 5, pp. 389-394, 2009.

[29] J. J. Faria, S. Krause, and J. Krause, "Collective behavior in road crossing pedestrians: the role of social information," Behavioral Ecology, vol. 21, no. 6, pp. 1236-1242, 2010.

[30] X. Xin, N. Jia, L. Zheng, and S. Ma, "Power-law in pedestrian crossing flow under the interference of vehicles at an unsignalized midblock crosswalk," Physica A: Statistical Mechanics and its Applications, vol. 406, pp. 287-297, 2014.

[31] L. Wang, S. Q. Ye, and N. G. Xie, "Crossing behavior of pedestrians and vehicles at unsignalized intersection based on dirty faces game," Acta Physica Sinica (Chinese Edition), vol. 64, no. 12, article 120201, 2015.

[32] R. A. Weber, "Behavior and learning in the "Dirty Faces" game," Experimental Economics, vol. 4, no. 3, pp. 229-242, 2001.

[33] R. C. Bayer and M. Chan, The dirty faces game revisited, School of Economics Working Papers, 2009.

[34] J. E. Littlewood, A Mathematician's Miscellany, Methuen \& Co., Ltd., London, UK, 1953. 
[35] W. Zhang, K. Wang, L. Wang, Z. Feng, and Y. Du, "Exploring factors affecting pedestrians' red-light running behaviors at intersections in China," Accident Analysis \& Prevention, vol. 96, pp. 71-78, 2016.

[36] J. Gou, X. Cai, and S. Dou, "Behavior simulation of "Chinese style road crossing" based on evolutionary game theory," in Proceedings of the International Conference on Logistics, Informatics and Service Sciences, 2017.

[37] N. Duduta, Q. Zhang, and M. Kroneberger, "Impact of intersection design on pedestrians' choice to cross on red," Transportation Research Record, vol. 2464, pp. 93-99, 2014.

[38] A. Dommes, M.-A. Granié, M.-S. Cloutier, C. Coquelet, and F. Huguenin-Richard, "Red light violations by adult pedestrians and other safety-related behaviors at signalized crosswalks," Accident Analysis \& Prevention, vol. 80, pp. 67-75, 2015.

[39] B. Li, "A model of pedestrians' intended waiting times for street crossings at signalized intersections," Transportation Research Part B: Methodological, vol. 51, pp. 17-28, 2013.

[40] S. Zöllner, "The impact of waiting time and other factors on dangerous pedestrian crossings and violations at signalized intersections: a case study in montreal," Transportation Research Part F: Traffic Psychology and Behaviour, vol. 21, no. 21, pp. 159172, 2013.

[41] S. Chandra and A. K. Bharti, "Speed distribution curves for pedestrians during walking and crossing," Procedia - Social and Behavioral Sciences, vol. 104, pp. 660-667, 2013. 


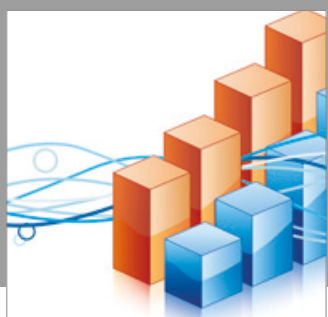

Advances in

Operations Research

vatersals

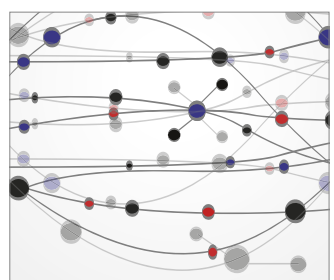

\section{The Scientific} World Journal
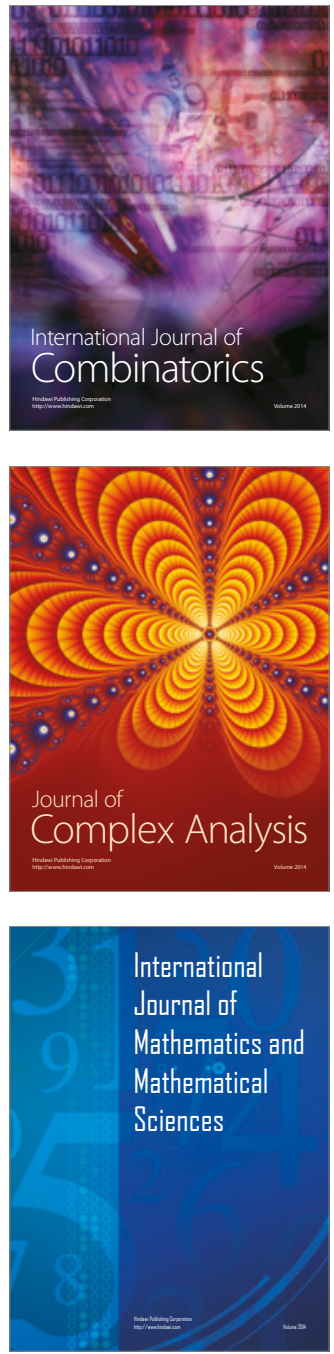
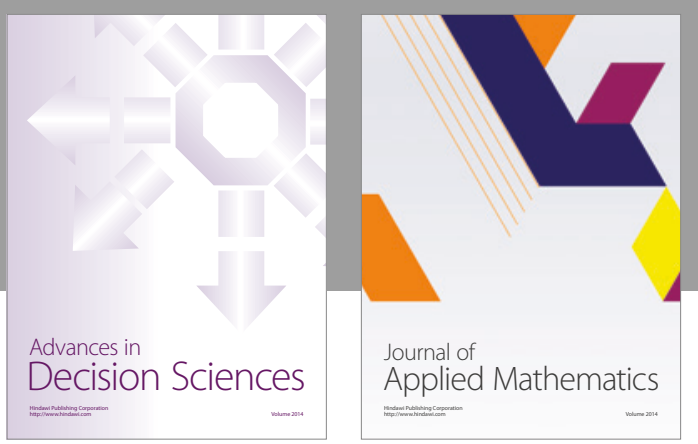

Algebra

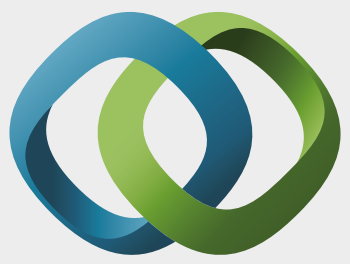

\section{Hindawi}

Submit your manuscripts at

https://www.hindawi.com
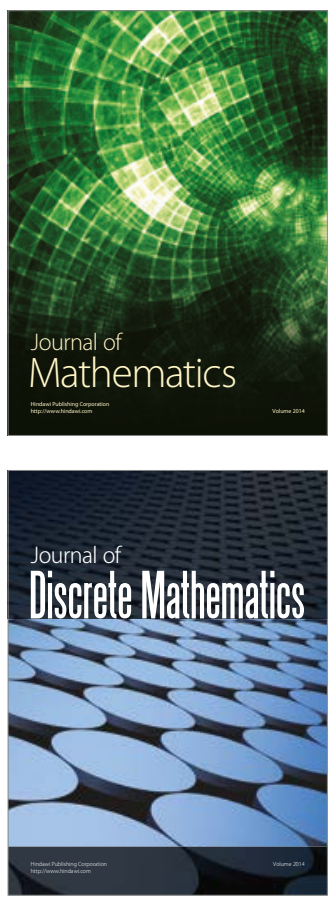

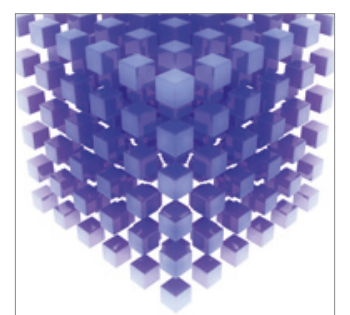

Mathematical Problems in Engineering
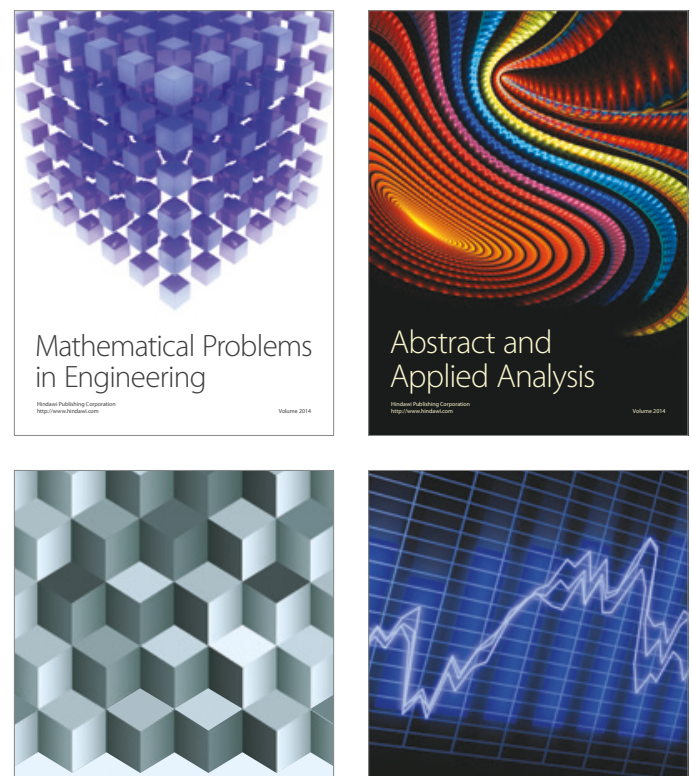

Journal of

Function Spaces

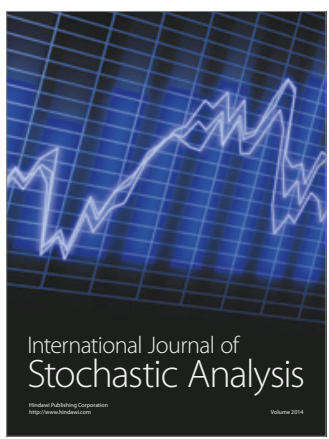

Probability and Statistics
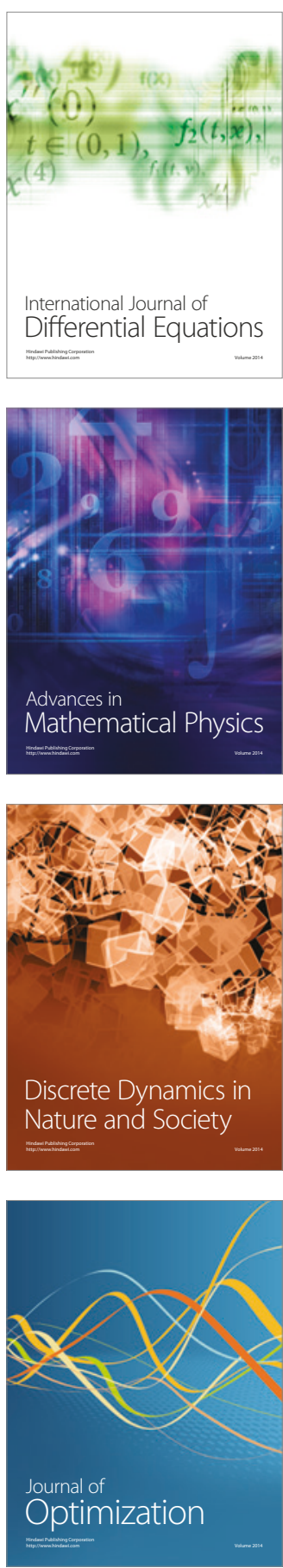